\title{
ON GLOBAL REGULARITY FOR SYSTEMS OF NONLINEAR WAVE EQUATIONS WITH THE NULL-CONDITION
}

\author{
CAN GAO, APARAJITA DASGUPTA, AND JOACHIM KRIEGER
}

\begin{abstract}
Aвstract. In this note we combine a recent result by Geba [2] on the local wellposedness theory of systems of nonlinear wave equations with $Q_{0}$ null-form structure with the classical Penrose compactification method to obtain a new small data global existence result with good point wise bounds.
\end{abstract}

\section{INTRODUCTION}

In this note we investigate the problem of global regularity for systems of nonlinear wave equations on Minkowski space $\mathbb{R}^{3+1}$ of the form

$$
\square u^{I}=\Gamma_{J K}^{I}(\vec{u}) \partial_{\alpha} u^{J} \partial^{\alpha} u^{K}, I=1,2 \ldots, N,
$$

where the index $\alpha$ gets summed over $\alpha=0,1, \ldots, 3$, and the indices $J, K$ are summed over $1, \ldots, N$. Equations of this type play a prominent role in the theory of nonlinear waves, due to the special cancellation structure inherent in them, namely the $Q_{0}$-null-form. This causes equations of this type to be amenable to better global regularity results than generic quadratic source terms, as witnessed for example in recent progress on the Wave Maps problem. The history of (1.1) goes at least back to the pivotal works [1], [5] by Christodoulou and Klainerman, respectively, which showed that in the physical space-dimension $n=3$, the nullcondition implies that smooth initial data $\left.\left(\vec{u}, \vec{u}_{t}\right)\right|_{t=0}$ that decay sufficiently fast and are suitably small result in global in time smooth solutions. Of course this encapsulates much more equations than those of the form (1.1), and in particular certain quasilinear ones. The methods in the cited papers differ markedly, with the second one relying on the commuting vector fields method, while the first one used the Penrose compactification method. Both Klainerman's and Christodoulou's method apparently require $(|x| \nabla)^{i} u$ to be small for $i=0,1, \ldots, 7$, and a natural if highly non-trivial question is to enquire about the optimal condition ensuring global existence of solutions as well as sharp point wise decay bounds, in terms of the decay and number of derivatives of the data at $|x|=\infty$. A recent result by Pusateri [6] furnishes much weaker conditions resulting in global existence and sharp decay bounds, and in particular imposes only $|x|^{2}$-weights on sufficiently many derivatives. His result relies on the recently developed 'space-time resonances' method, see e. g. [7].

Our goal here is to explore a somewhat different approach to this problem, namely the combination of the Penrose compactification approach with a new local 
well-posedness result for nonlinear waves on curved backgrounds by Geba [2] and Geba-Tataru [3]. The latter has its origins in the harmonic analysis approach to optimal local well-posedness, such as [8]. Specifically, we prove the following

Theorem 1.1. Let $s>\frac{3}{2}$, and assume that the functions $\Gamma_{J K}^{I}(\cdot) \in C^{\infty}(\mathbb{R})$ with globally bounded derivatives. Further, assume the data $\left(u_{0}, u_{1}\right)=\left.\left(u, u_{t}\right)\right|_{t=0} \in$ $C_{0}^{\infty}\left(\mathbb{R}^{3}\right) \times C_{0}^{\infty}\left(\mathbb{R}^{3}\right)$ satisfy the smallness condition

$$
\left\|\langle x\rangle^{2 s-1} u_{0}\right\|_{H^{s}}+\left\|\langle x\rangle^{2 s-1} u_{1}\right\|_{H^{s-1}}<\varepsilon
$$

for some sufficiently small constant $\varepsilon=\varepsilon\left(s, \Gamma_{J K}^{I}\right)>0$. Then there exists a global smooth solution for (1.1) with these initial data. Moreover, this solution satisfies the point wise bounds

$$
|u(t, x)| \leqslant C(1+|| t|-| x||)^{-1}(1+|| t|+| x||)^{-1}
$$

Remark 1.1. We note that the global existence assertion can also be inferred from the result [8], which however uses quite sophisticated spaces at the critical scaling regularity. The precise point wise bound here, on the other hand, appears to be a genuine consequence of the method of this paper.

\section{ACKNOWLEDGEMENT}

The authors would like to thank W. Schlag and W. Wong for helpful comments and to $\mathrm{W}$. Wong for pointing out an error in an earlier version.

\section{TeChNiCAL PRELIMINARIES}

We record the following lemma, which will be essential to run an interpolation argument; denote $\langle\nabla\rangle=\sqrt{1-\triangle}$. Then

Lemma 2.1. Let $u \in C_{0}^{\infty}\left(\mathbb{R}^{3}\right)$. Then we have for any $s \in \mathbb{R}$

$$
\begin{aligned}
& \sum_{0 \leqslant|\alpha| \leqslant 2}\left\|\langle x\rangle^{3} \nabla^{\alpha} u\right\|_{L_{x}^{2}} \lesssim\langle s\rangle^{2}\left\|\langle\nabla\rangle^{2}\left(\langle x\rangle^{3+i s} u\right)\right\|_{L_{x}^{2}}, \\
& \sum_{0 \leqslant|\alpha| \leqslant 1}\left\|\langle x\rangle \nabla^{\alpha} u\right\|_{L_{x}^{2}} \lesssim\langle s\rangle\left\|\langle\nabla\rangle\left(\langle x\rangle^{1+i s} u\right)\right\|_{L_{x}^{2}}
\end{aligned}
$$

Proof. We have

$$
\left\|\nabla^{2}\left(\langle x\rangle^{3+i s} u\right)\right\|_{L_{x}^{2}} \geqslant\left\|\langle x\rangle^{3} \nabla^{2} u\right\|_{L_{x}^{2}}-2\left\|\nabla\left(\langle x\rangle^{3+i s}\right) \nabla u\right\|_{L_{x}^{2}}-\left\|\nabla^{2}\left(\langle x\rangle^{3+i s}\right) u\right\|_{L_{x}^{2}}
$$

Further, we have

$$
\begin{aligned}
\left\|\langle x\rangle^{2} \nabla u\right\|_{L_{x}^{2}}=\left\|\langle x\rangle^{-1}\langle x\rangle^{3+i s} \nabla u\right\|_{L_{x}^{2}} & \lesssim\left\|\langle x\rangle^{-1} \nabla\left(\langle x\rangle^{3+i s} u\right)\right\|_{L_{x}^{2}}+\langle s\rangle\|\langle x\rangle u\|_{L_{x}^{2}} \\
& \lesssim\left\|\nabla^{2}\left(\langle x\rangle^{3+i s} u\right)\right\|_{L_{x}^{2}}+\langle s\rangle\|\langle x\rangle u\|_{L_{x}^{2}},
\end{aligned}
$$

where in the last step we have used Hardy's inequality. It follows that

$$
\left\|\nabla\left(\langle x\rangle^{3+i s}\right) \nabla u\right\|_{L_{x}^{2}}+\left\|\nabla^{2}\left(\langle x\rangle^{3+i s}\right) u\right\|_{L_{x}^{2}} \lesssim\langle s\rangle^{2}\left\|\langle\nabla\rangle^{2}\left(\langle x\rangle^{3+i s} u\right)\right\|_{L_{x}^{2}}
$$


Further, we have

$$
\left\|\langle x\rangle^{3} \nabla u\right\|_{L_{x}^{2}}=\left\|\langle x\rangle^{3+i s} \nabla u\right\|_{L_{x}^{2}} \lesssim\left\|\nabla\left(\langle x\rangle^{3+i s} u\right)\right\|_{L_{x}^{2}}+\langle s\rangle\left\|\langle x\rangle^{2} u\right\|_{L_{x}^{2}}
$$

The first inequality of the lemma follows from (2.1) - (2.3), and the second inequality of the lemma is more of the same.

\section{Penrose Compactification}

Here we quickly recall the Penrose Compactification of $\mathbb{R}^{3+1}$, as expounded in Hormander [4]. Let $\mathbf{E}^{1+3}$ be the Einstein cylinder given by

$$
\mathbf{E}^{1+3}:=(-\pi, \pi) \times S^{3},
$$

and let $\overline{\mathbf{E}}^{1+3} \subset \mathbf{E}^{1+3}$ the set defined by

$$
\overline{\mathbf{E}}^{1+3}:=\{(T, \cos R, \omega \sin R):-\pi<T<\pi, 0 \leqslant R<\pi, R+|T|<\pi\}
$$

Using spherical coordinates $(r, \omega), r=|x|$, on $\mathbb{R}^{n}$, define the map $\Psi: \mathbb{R}^{1+3} \longrightarrow$ $\overline{\mathbf{E}}^{1+3}$ given by

$$
T=\tan ^{-1}(t+r)+\tan ^{-1}(t-r), R=\tan ^{-1}(t+r)-\tan ^{-1}(t-r),
$$

with inverse $\Psi^{-1}: \overline{\mathbf{E}}^{1+3} \longrightarrow \mathbb{R}^{1+3}$ given by

$$
(T, X) \longrightarrow \frac{1}{\cos T+X_{0}}(\sin T, \vec{X}) ;
$$

Here we use coordinates $X=\left(\begin{array}{c}X_{0} \\ X_{1} \\ X_{2} \\ X_{3}\end{array}\right)$ on $\mathbb{R}^{4}$. Now cover $S^{3}$ by two open sets $U, V$, with $U$ and $V$ containing the north pole, respectively the south pole $N:=\left(\begin{array}{c} \pm 1 \\ 0 \\ 0 \\ 0\end{array}\right)$. Using stereographic projection from $S$ to $\mathbb{R}^{3}$ to introduce coordinates $Y$ on $U$, we can write

$$
X_{0}=\frac{1-Y^{2}}{1+Y^{2}}, \sin R \omega=\frac{2 Y}{1+Y^{2}}
$$

In fact, by choosing $U$ suitably, we may arrange that $Y$ ranges over a bounded subset of $\mathbb{R}^{3}$; similar considerations apply to $V$. We immediately observe that the initial data hyperplane $t=0$ corresponds to $T=0, R=2 \tan ^{-1} r \in[0, \pi)$, whence $X_{0}=\cos R=\frac{1-r^{2}}{1+r^{2}}$. In order to translate the original equations (1.1) into a problem on $(-\pi, \pi) \times S^{3}$, following Hormander, we introduce the new variables

$$
\tilde{u}^{I}:=\Omega(T, X)^{-1} u^{I}\left(\Psi^{-1}(T, X)\right), I=1,2, \ldots, N,
$$

where we use the notation

$$
\Omega(T, X):=\cos T+X_{0}
$$


We note that the vector fields $X_{k} \frac{\partial}{\partial X_{j}}-X_{j} \frac{\partial}{\partial X_{k}}, k, j$ ranging over $0,1,2,3$, together with $\frac{\partial}{\partial T}$ generate the tangent space at each point of $(-\pi, \pi) \times S^{3}$. Restricting to $T=0$, only the vector fields $X_{k} \frac{\partial}{\partial X_{0}}-X_{0} \frac{\partial}{\partial X_{k}}$ have a nonzero effect on the outer factor $\Omega(T, X)^{-1}$, and we have

$$
\left.\left(X_{k} \frac{\partial}{\partial X_{0}}-X_{0} \frac{\partial}{\partial X_{k}}\right)^{\alpha}\left(\Omega(T, X)^{-1}\right)\right|_{0 \times S^{3}}=O\left(\Omega^{-1-\frac{\alpha}{2}}(T, X)\right), \alpha=0,1,2 .
$$

Using this we can infer the following

Lemma 3.1. Using the preceding notation, we have for $\operatorname{Re} \alpha=0,1,2$

$$
\sum_{j, k=0}^{3}\left\|\left(X_{k} \frac{\partial}{\partial X_{j}}-X_{j} \frac{\partial}{\partial X_{k}}\right)^{\operatorname{Re} \alpha} \tilde{u}^{I}\right\|_{L^{2}\left(0 \times S^{3}\right)} \lesssim\langle\operatorname{Im} \alpha\rangle^{2}\left\|\left\langle\nabla_{x}\right\rangle^{\alpha}\left(\langle x\rangle^{2 \alpha-1} u^{I}\right)\right\|_{L^{2}\left(0 \times \mathbb{R}^{3}\right)}
$$

Proof. Let $\operatorname{Re} \alpha=2$. When both derivatives fall on the outer factor $\Omega^{-1}$, using the observation above, we have to estimate the expression

$$
\int_{0 \times S^{3}} \Omega^{-4}\left(u^{I}\right)^{2}\left(\Psi^{-1}(T, X)\right) d \sigma=\int_{0 \times \mathbb{R}^{3}} \Omega^{-1}\left(u^{I}\right)^{2} d x,
$$

using the fact that $d T^{2}-d X^{2}=\Omega^{2}\left(d t^{2}-d x^{2}\right)$, see Hormander [4]. Since we have

$$
\Omega(0, X)=\frac{2}{1+r^{2}},
$$

we can bound the preceding by $\left\|\langle x\rangle u^{I}\right\|_{L^{2}\left(0 \times \mathbb{R}^{3}\right)}^{2}$. In case both derivatives fall on the factor $u^{I}$, using the fact (see Hormander [4]) that

$$
X_{0} \partial_{X_{k}}-X_{k} \partial_{X_{0}}=\frac{1}{2}\left(1+t^{2}-|x|^{2}\right) \partial_{x_{k}}+x_{k}\left(t \partial_{t}+\left\langle x, \partial_{x}\right\rangle\right)
$$

we obtain the expression

$$
\int_{0 \times \mathbb{R}^{3}} \Omega^{-2}\langle x\rangle^{8}\left|\left\langle\nabla_{x}\right\rangle^{2} u^{I}\right|^{2} \Omega^{3} d x \lesssim \int_{0 \times \mathbb{R}^{3}}\langle x\rangle^{6}\left|\left\langle\nabla_{x}\right\rangle^{2} u^{I}\right|^{2} d x
$$

The case when one derivative falls on $\Omega^{-1}$ leads to a similar bound. Using Lemma 2.1 furnishes the desired bound.

Applying the Stein interpolation theorem to the family of operators indexed by $S$

$$
v^{I} \longrightarrow\left(\frac{\widetilde{v^{I}}}{\langle x\rangle^{2 s-1}}\right)
$$

and which for Re $s=1$ maps $H^{1}\left(0 \times \mathbb{R}^{3}\right)$ into $H^{1}\left(0 \times S^{3}\right)$, while for $\operatorname{Re} s=2$ it maps $H^{2}\left(0 \times \mathbb{R}^{3}\right)$ into $H^{2}\left(0 \times S^{3}\right)$, we obtain that

$$
\left\|\tilde{u}^{I}\right\|_{H^{s}\left(0 \times S^{3}\right)} \lesssim\left\|\langle x\rangle^{2 s-1} u^{I}\right\|_{H^{s}\left(0 \times \mathbb{R}^{3}\right)},
$$

provided $1 \leqslant s \leqslant 2$.

As for the time derivative $\left.\partial_{T} \tilde{u}^{I}\right|_{T=0}$, Hormander [4] furnishes the formula

$$
\partial_{T}=\frac{1}{2}\left(1+t^{2}+|x|^{2}\right) \partial_{t}+t\left\langle x, \partial_{x}\right\rangle
$$


and so an argument just like the preceding furnishes the bound

$$
\left\|\left.\partial_{T} \tilde{u}^{I}\right|_{T=0}\right\|_{H^{s-1}\left(0 \times S^{3}\right)} \lesssim\left\|\langle x\rangle^{2 s-1} \partial_{t} u^{I}\right\|_{H^{s-1}\left(0 \times \mathbb{R}^{3}\right)}
$$

for the same range of $s$.

In order to invoke the theory developed by Geba-Tataru [3], we need to work in the context of $\mathbb{R}^{3}$. For that purpose, we localize the functions $\tilde{u}^{I}$ to one of the stereographic coordinate charts $U, V$. Thus denoting by $\chi_{1,2}$ a smooth partition of unity subordinate to $U, V$, we have

$\left\|\chi_{1} \tilde{u}^{I}\right\|_{H_{Y}^{s}\left(\mathbb{R}^{3}\right)}+\left\|\chi_{1} \partial_{T} \tilde{u}^{I}\right\|_{H_{Y}^{s-1}\left(\mathbb{R}^{3}\right)}\|\lesssim\|\langle x\rangle^{2 s-1} u^{I}\left\|_{H^{s}\left(0 \times \mathbb{R}^{3}\right)}+\right\|\langle x\rangle^{2 s-1} \partial_{t} u^{I} \|_{H^{s-1}\left(0 \times \mathbb{R}^{3}\right)}$,

with a similar estimate for the truncation of $\tilde{u}^{I}$ to $V$.

\section{THE EQUATIONS IN STEREOGRAPHIC COORDINATES}

Here we express the system (1.1) with respect to the coordinates $Y$, showing that this is indeed of the form handled by the result in [2]. According to the above, applying the Penrose transform we get a system as follows

$$
\left(\tilde{\square}_{g}+1\right) \tilde{u}^{I}=\Gamma_{J K}^{I}(\Omega \tilde{u}) \Omega^{-3} \tilde{F}
$$

here $\tilde{F}$ is given as

$$
\tilde{F}=\left\langle\nabla\left(\Omega \tilde{u}^{J}\right), \nabla\left(\Omega \tilde{u}^{K}\right)\right\rangle
$$

and the inner product of vectors $\langle$,$\rangle is defined with respect to the Minkowski met-$ ric. Here we will have to deal with the three terms just from a direct expansion of $\tilde{F}$, which are $\langle\nabla \Omega, \nabla \Omega\rangle,\left\langle\nabla \Omega, \nabla \tilde{u}^{J, K}\right\rangle,\left\langle\nabla \tilde{u}^{J}, \nabla \tilde{u}^{K}\right\rangle$.

To make the calculation looks a bit simpler, we let

$$
X_{0}=\frac{1-|Y|^{2}}{1+|Y|^{2}}, \quad \Sigma^{J}=\sum_{i=1}^{3} Y_{i} \partial_{Y_{i}} \tilde{u}^{J}
$$

First we will deal with the easier terms such as $\langle\nabla \Omega, \nabla \Omega\rangle,\left\langle\nabla \Omega, \nabla \tilde{u}^{J, K}\right\rangle$.

$$
\begin{aligned}
\langle\nabla \Omega, \nabla \Omega\rangle & =\Omega^{2} X_{0}^{2} \sin ^{2} T-\Omega^{2}\left(1-X_{0}^{2}\right) \cos ^{2} T \\
& =-\Omega^{2} \cos ^{2} T+\Omega^{2} X_{0}^{2} \\
& =\Omega^{2}\left(X_{0}^{2}-\cos ^{2} T\right)
\end{aligned}
$$

We use $U$ to formally replace $\tilde{u}^{J, K}$ and $U_{i}$ stands for the derivatives of space-time.

$$
\begin{aligned}
\langle\nabla \Omega, \nabla U\rangle & =-\Omega X_{0}\left(1+X_{0} \cos T\right) \sin T U_{0}+\Omega X_{0} \sin ^{2} T \Sigma-\Omega\left(1-X_{0}^{2}\right) \cos T \sin T U_{0} \\
& +\Omega\left(1+X_{0}\right) \cos T(1+\cos T) \Sigma-\Omega\left(\cos ^{2} T+X_{0} \cos T\right) \Sigma \\
& =-\Omega \sin T\left(X_{0}+\cos T\right) U_{0}+\Omega\left(\cos T+X_{0}\right) \Sigma \\
& =\Omega\left[\left(\cos T+X_{0}\right)\left(\Sigma-\sin T U_{0}\right)\right]
\end{aligned}
$$


Next we calculate $\left\langle\nabla \tilde{u}^{J}, \nabla \tilde{u}^{K}\right\rangle$.

$\left\langle\nabla \tilde{u}^{J}, \nabla \tilde{u}^{K}\right\rangle=\left[\left(1+X_{0} \cos T\right) \tilde{u}_{0}^{J}-\sin T \Sigma^{J}\right]\left[\left(1+X_{0} \cos T\right) \tilde{u}_{0}^{K}-\sin T \Sigma^{K}\right]-\sum_{i=1}^{3} V_{i}^{J} M_{i}\left(V_{i}^{K}\right)^{T}$

where $V_{i}^{J}=\left(\Sigma^{J}, \tilde{u}_{0}^{J}, \tilde{u}_{i}^{J}\right)$ and the matrix $M_{i}$ is defined as

$$
\left(\begin{array}{ccc}
A_{i} A_{i} & B_{i} A_{i} & C A_{i} \\
A_{i} B_{i} & B_{i} B_{i} & C B_{i} \\
A_{i} C & B_{i} C & C^{2}
\end{array}\right)
$$

where

$$
A_{i}=(1-\cos T) Y_{i}, \quad B_{i}=-\frac{2 \sin T Y_{i}}{1+|Y|^{2}}, \quad C=\frac{1}{2}\left(\left(1+|Y|^{2}\right) \cos T+1-|Y|^{2}\right)
$$

A direct calculation gives us that

$$
\begin{aligned}
\sum_{i=1}^{3} V_{i}^{J} M_{i}\left(V_{i}^{K}\right)^{T} & =(1-\cos T)^{2}|Y|^{2} \Sigma^{J} \Sigma^{K}-\left(1-X_{0}\right) \sin T(1-\cos T)\left(\Sigma^{J} \tilde{u}_{0}^{K}+\Sigma^{K} \tilde{u}_{0}^{J}\right) \\
& +\left(1-X_{0}^{2}\right) \sin ^{2} T \tilde{u}_{0}^{J} \tilde{u}_{0}^{K}+\sum_{i=1}^{3} C A_{i}\left(\Sigma^{J} \tilde{u}_{i}^{K}+\Sigma^{K} \tilde{u}_{i}^{J}\right)+\sum_{i=1}^{3} B_{i} C\left(\tilde{u}_{0}^{J} \tilde{u}_{i}^{K}+\tilde{u}_{0}^{K} \tilde{u}_{i}^{J}\right) \\
& +\sum_{i=1}^{3} C^{2} \tilde{u}_{i}^{J} \tilde{u}_{i}^{K}
\end{aligned}
$$

Further more we get

$$
\sum_{i=1}^{3} C A_{i}\left(\Sigma^{J} \tilde{u}_{i}^{K}+\Sigma^{K} \tilde{u}_{i}^{J}\right)=2 C(1-\cos T) \Sigma^{J} \Sigma^{K}
$$

and

$$
\sum_{i=1}^{3} B_{i} C\left(\tilde{u}_{0}^{J} \tilde{u}_{i}^{K}+\tilde{u}_{0}^{K} \tilde{u}_{i}^{J}\right)=-\frac{2 \sin T C}{1+|Y|^{2}}\left(\tilde{u}_{0}^{J} \Sigma^{K}+\tilde{u}_{0}^{K} \Sigma^{J}\right)
$$

Then from (4.1), (4.2), (4.3), (4.4) we have so far

$$
\left\langle\nabla \tilde{u}^{J}, \nabla \tilde{u}^{K}\right\rangle=\left(\cos T+X_{0}\right)^{2}\left[\tilde{u}_{0}^{J} \tilde{u}_{0}^{K}-\frac{\left(1+|Y|^{2}\right)^{2}}{4} \sum_{i=1}^{3} \tilde{u}_{i}^{J} \tilde{u}_{i}^{K}\right]
$$

Finally we calculate $\tilde{F}$ as

$$
\begin{aligned}
\tilde{F} & =\Omega^{2}\left[\left(X_{0}^{2}-\cos ^{2} T\right) \tilde{u}^{J} \tilde{u}^{K}-\left(\cos T+X_{0}\right)\left[\tilde{u}^{K}\left(\sin T \tilde{u}_{0}^{J}-\Sigma^{J}\right)+\tilde{u}^{J}\left(\sin T \tilde{u}_{0}^{K}-\Sigma^{k}\right)\right]\right. \\
& \left.+\left(\cos T+X_{0}\right)^{2}\left[\tilde{u}_{0}^{J} \tilde{u}_{0}^{K}-\frac{\left(1+|Y|^{2}\right)^{2}}{4} \sum_{i=1}^{3} \tilde{u}_{i}^{J} \tilde{u}_{i}^{K}\right]\right]
\end{aligned}
$$


We then have the transformed equation as

$$
\begin{aligned}
& \left(\tilde{\square}_{g}+1\right) \tilde{u}^{I} \\
& =\Gamma_{J K}^{I}(\Omega \tilde{u}) \Omega^{-1}\left[\left(X_{0}^{2}-\cos ^{2} T\right) \tilde{u}^{J} \tilde{u}^{K}\right. \\
& -\left(\cos T+X_{0}\right)\left[\tilde{u}^{K}\left(\sin T \tilde{u}_{0}^{J}-\Sigma^{J}\right)+\tilde{u}^{J}\left(\sin T \tilde{u}_{0}^{K}-\Sigma^{k}\right)\right] \\
& \left.+\left(\cos T+X_{0}\right)^{2}\left[\tilde{u}_{0}^{J} \tilde{u}_{0}^{K}-\frac{\left(1+|Y|^{2}\right)^{2}}{4} \sum_{i=1}^{3} \tilde{u}_{i}^{J} \tilde{u}_{i}^{K}\right]\right]
\end{aligned}
$$

\section{LOCAL WELL-POSEDNESS OF THE TRANSFORMED SYSTEM}

We observe that the transformed equations take the following schematic form:

$$
\left(\tilde{\square}_{g}+1\right) \tilde{u}^{I}=\Gamma_{J K}^{I}(\Omega \tilde{u})\left[A^{J K}+B^{J K}+C^{J K}\right],
$$

where we have

$$
\begin{gathered}
A^{J K}=\left(\frac{1-Y^{2}}{1+Y^{2}}-\cos T\right) \tilde{u}^{J} \tilde{u}^{K}, \\
B^{J K}=\tilde{u}^{J}\left(\sum_{j} Y_{j} \partial_{Y_{j}} \tilde{u}^{K}-\sin T \partial_{T} \tilde{u}^{K}\right) \\
C^{J K}=\left(\cos T+\frac{1-Y^{2}}{1+Y^{2}}\right)\left(\partial_{T} \tilde{u}^{J} \partial_{T} \tilde{u}^{K}-\frac{\left(1+|Y|^{2}\right)^{2}}{4} \sum_{i=1}^{3} \partial_{Y_{i}} \tilde{u}^{J} \partial_{Y_{i}} \tilde{u}^{K}\right) \\
=\left(\cos T+\frac{1-Y^{2}}{1+Y^{2}}\right) Q_{0}^{g}\left(\tilde{u}^{J}, \tilde{u}^{K}\right)
\end{gathered}
$$

where $g$ is the metric on $(-\pi, \pi) \times S^{3}$ expressed with respect to the local coordinates $(T, Y)$.

Our task is to prove local well-posedness of (5.1) in $H^{s}\left(\mathbb{R}^{3}\right)$ and data supported in a fixed compact set, since then Theorem 1.1 follows by invoking (3.3) as well as the Huyghen's principle to glue the local solutions together. The local well-posedness follows essentially immediately from the functional framework developed in [2], except that we need to take some additional factors and error terms into account.

5.1. Function spaces. Here we quickly recall the norms used in [3], [2]. For $s>\frac{3}{2}$ and $\frac{1}{2}<\theta<s-1$, we introduce on $L^{2}\left([-\pi, \pi] \times \mathbb{R}^{3}\right)$ the norms

$$
\|\tilde{u}\|_{X^{s, \theta}}^{2}:=\inf \left\{\sum_{\lambda=1}^{\infty} \sum_{d=1}^{\lambda}\left\|\tilde{u}_{\lambda, d}\right\|_{X_{\lambda, d}^{s, \theta}}^{2} ; \sum_{\lambda=1}^{\infty} \sum_{d=1}^{\lambda} S_{\lambda} \tilde{u}_{\lambda, d}=\tilde{u}\right\}
$$

Here the operators $S_{\lambda}$ localize to dyadic spatial frequency $\lambda$ for $\lambda>1$, and to frequency $<1$ for $\lambda=1$. The numbers $d$ are dyadic as well. Also, the norms $\|\cdot\|_{X_{\lambda, d}^{s, \theta}}$ are defined by

$$
\|\tilde{u}\|_{X_{\lambda, d}^{s, \theta}}=\lambda^{s} d^{\theta}\|\tilde{u}\|_{L^{2}}+\lambda^{s-1} d^{\theta-1}\left\|\square_{S_{<\sqrt{\lambda}} \tilde{u}} \tilde{u}\right\|_{L^{2}},
$$

where $L^{2}=L^{2}\left([-\pi, \pi] \times \mathbb{R}^{3}\right)$, and we can use the spatially cut-off $S_{<\sqrt{\lambda} g}$ as opposed to the space-time variant in [2] since the metric in our case does not depend 
on $T$. As indicated in the notation, theses norms are used to control the functions $\tilde{u}^{J}$.

We also have the spaces $X^{s-1, \theta-1}$, used to control the source terms on the right:

$\|f\|_{X^{s-1, \theta-1}}^{2}:=\inf \left\{\left\|f_{0}\right\|_{L^{2} H^{s-1}}^{2}+\sum_{\lambda=1}^{\infty} \sum_{d=1}^{\lambda}\left\|f_{\lambda, d}\right\|_{X_{\lambda, d}^{s, \theta}}^{2} ; f=f_{0}+\sum_{\lambda=1}^{\infty} \sum_{d=1}^{\lambda} \square_{S_{<\sqrt{\lambda}} g} S_{\lambda} f_{\lambda, d}\right\}$

5.2. Estimating the source terms. Recall that we may assume the coordinates $Y$ to be restricted to a compact set. This means that we can replace the factors

$$
\cos T+\frac{1-Y^{2}}{1+Y^{2}}
$$

by the smooth compactly supported function

$$
\chi(Y, T)\left[\cos T+\frac{1-Y^{2}}{1+Y^{2}}\right]
$$

for a suitable cutoff $\chi(T, Y) \in C_{0}^{\infty}\left(\mathbb{R}^{3+1}\right)$. Then use the simple

Lemma 5.1. For $\phi \in C_{0}^{\infty}\left(\mathbb{R}^{3+1}\right)$, we have

$$
\|\phi\|_{X^{s, \theta}}<\infty
$$

Proof. Write $\phi=\sum_{\lambda=1}^{\infty} S_{\lambda} \phi$, as well as $d=1$. Then

$$
\left\|S_{\lambda} \phi\right\|_{X_{\lambda, 1}^{s, \theta}} \lesssim_{N} \lambda^{-N}
$$

for any $N$, since $S_{\lambda} \phi$ decays to any polynomial power in $\lambda^{-1}$. The lemma follows from this.

Replacing the right hand side of (5.1) by

$$
\chi(T, Y) \Gamma_{J K}^{I}(\Omega \tilde{u})\left[A^{J K}+B^{J K}+C^{J K}\right]
$$

for a smooth cutoff $\chi(T, Y) \in C_{0}^{\infty}\left(\mathbb{R}^{3+1}\right)$, we can use Proposition 3.3 in [2] to conclude that

$$
\left\|\chi(T, Y) \Gamma_{J K}^{I}(\Omega \tilde{u})\right\|_{X^{s, \theta}} \leqslant C\left(\|\tilde{u}\|_{L^{\infty}}\right)\left(1+\|\tilde{u}\|_{X^{s, \theta}}^{5}\right)
$$

Also, note that due to Lemma 2.4 in [2], as well as Sobolev's inequality, we have

$$
\|\tilde{u}\|_{L^{\infty}} \lesssim\|\tilde{u}\|_{X^{s, \theta}}
$$

under our assumptions on $s, \theta$. We now estimate the source terms as follows:

I: Estimate for $A^{J K}$. We have

$$
\begin{aligned}
\left\|\chi(Y, T) A^{J K}\right\|_{X^{s-1, \theta-1}} & \leqslant\left\|\chi(Y, T) A^{J K}\right\|_{L^{2} H^{s-1}} \\
& \lesssim\left\|\chi(Y, T)\left(\frac{1-Y^{2}}{1+Y^{2}}-\cos T\right)\right\|_{L_{t}^{\infty} H^{s}} \prod_{i=J, K}\left\|\tilde{u}^{i}\right\|_{L_{t}^{\infty}} H^{s} \\
& \lesssim \prod_{i=J, K}\left\|\tilde{u}^{i}\right\|_{X^{s, \theta}},
\end{aligned}
$$


where in the last step we have again used Lemma 2.4 in [2], and so

$$
\begin{aligned}
\left\|\chi(T, Y) \Gamma_{J K}^{I}(\Omega \tilde{u}) A^{J K}\right\|_{X^{s-1, \theta-1}} & \lesssim\left\|\tilde{\chi}(T, Y) \Gamma_{J K}^{I}(\Omega \tilde{u})\right\|_{X^{s, \theta}}\left\|\chi(Y, T) A^{J K}\right\|_{X^{s-1, \theta-1}} \\
& \lesssim C_{1}\left(\|\tilde{u}\|_{X^{s, \theta}}\right) \prod_{i=J, K}\left\|\tilde{u}^{i}\right\|_{X^{s, \theta}}
\end{aligned}
$$

where the compactly supported cutoff $\tilde{\chi}$ is chosen so that $\tilde{\chi} \chi=\chi$. Here we have taken advantage of Proposition 3.3 in [2] in the last inequality above.

II: Estimate for $B^{J K}$. Here we have, using the easily verified inequalities

$$
\|f \nabla g\|_{H^{s-1}} \lesssim\|f\|_{H^{s}}\|g\|_{H^{s}},\left\|f \partial_{T} g\right\|_{H^{s-1}} \lesssim\|f\|_{H^{s}}\left\|\partial_{T} g\right\|_{H^{s-1}}
$$

valid for $s>\frac{3}{2}$, that

$$
\begin{aligned}
\left\|\chi(T, Y) B^{J K}\right\|_{X^{s-1, \theta-1}} & \lesssim\left\|\chi(T, Y) \tilde{u}^{J}\right\|_{L_{t}^{2} H_{x}^{s}}\left\|\nabla_{T, Y} \tilde{u}^{K}\right\|_{L_{t}^{\infty} H_{x}^{s-1}} \\
& \lesssim \prod_{i=J, K}\left\|\tilde{u}^{i}\right\|_{X^{s, \theta}},
\end{aligned}
$$

where we have used that (Lemma 2.4 in [2])

$$
\left\|\nabla_{T, Y} \tilde{u}\right\|_{L_{t}^{\infty} H^{s-1}} \lesssim\|\tilde{u}\|_{X^{s, \theta}}
$$

Hence we again obtain

$$
\left\|\chi(T, Y) \Gamma_{J K}^{I}(\Omega \tilde{u}) B^{J K}\right\|_{X^{s-1, \theta-1}} \lesssim \prod_{i=J, K}\left\|\tilde{u}^{i}\right\|_{X^{s, \theta}},
$$

using the preceding lemma.

III: Estimate for $C^{J K}$. Here we use that

$$
\left\|Q_{0}^{g}\left(\tilde{u}^{J}, \tilde{u}^{K}\right)\right\|_{X^{s-1, \theta-1}} \lesssim \prod_{i=J, K}\left\|\tilde{u}^{i}\right\|_{X^{s, \theta}}
$$

see Remark 1.4 in [2]. We conclude that

$$
\begin{aligned}
\left\|\chi(T, Y) \Gamma_{J K}^{I}(\Omega \tilde{u}) Q_{0}^{g}\left(\tilde{u}^{J}, \tilde{u}^{K}\right)\right\|_{X^{s-1, \theta-1}} & \lesssim\left\|\chi(T, Y) \Gamma_{J K}^{I}(\Omega \tilde{u})\right\|_{X^{s, \theta}}\left\|Q_{0}^{g}\left(\tilde{u}^{J}, \tilde{u}^{K}\right)\right\|_{X^{s-1, \theta-1}} \\
& \lesssim \prod_{i=J, K}\left\|\tilde{u}^{i}\right\|_{X^{s, \theta}}
\end{aligned}
$$

Using I - III, we can now immediately infer

Proposition 5.2. Let $\left(\tilde{u}_{0}, \tilde{u}_{1}\right)=\left(\left(\tilde{u}_{0}^{I}\right),\left(\tilde{u}_{1}^{I}\right)\right),\left(\tilde{u}_{0}^{I}, \tilde{u}_{1}^{I}\right) \in\left(H^{s} \times H^{s-1}\right)\left(\mathbb{R}^{3}\right) \forall I, s>\frac{3}{2}$, be supported within the fixed compact set $K \subset \mathbb{R}^{3}$. Then there exists a time $T=$ $T\left(\left\|\left(\tilde{u}_{0}, \tilde{u}_{1}\right)\right\|_{H^{s} \times H^{s-1}}, K\right)>0$ such that (5.1) admits a unique solution $\tilde{u}=\left(\tilde{u}^{I}\right)$, $\tilde{u}^{I} \in C^{1}\left([-T, T], H^{s}\left(\mathbb{R}^{3}\right)\right)$ with initial data $\left(\tilde{u}_{0}, \tilde{u}_{1}\right)$ and with $\tilde{u}_{t}^{I} \in C^{0}\left([-T, T], H^{s-1}\left(\mathbb{R}^{3}\right)\right)$ for each I. Moreover, there is some $T_{0}=T_{0}(K)>0$ and a $\epsilon_{0}>0$ such that if

$$
\left\|\left(\tilde{u}_{0}, \tilde{u}_{1}\right)\right\|_{H^{s} \times H^{s-1}}<\epsilon_{0},
$$

then local solution extends to the interval $\left[-T_{0}, T_{0}\right]$, and satisfies a bound

$$
\left\|\left(\tilde{u}(t, \cdot), \tilde{u}_{T}(t, \cdot)\right)\right\|_{H^{s} \times H^{s-1}} \leqslant C(K)\left\|\left(\tilde{u}_{0}, \tilde{u}_{1}\right)\right\|_{H^{s} \times H^{s-1}}, \forall t \in\left[-T_{0}, T_{0}\right] .
$$

If $\left(\tilde{u}_{0}, \tilde{u}_{1}\right) \in H^{s_{1}} \times H^{s_{1}-1}$ for $s_{1}>s$, the additional regularity gets preserved. 
We note that the statement concerning higher regularity follows in standard fashion from differentiating the equations. Also, observe that the coefficients and metric depend nontrivially on $Y$, whence even for small data, we only expect existence on a small time interval, as the solution may then escape from the particular coordinate patch, meaning $Y \rightarrow \infty$ in finite time.

\section{Proof of Theorem 1.1}

Cover the sphere $S^{3}$ with two open sets $U, V$ with $\bar{U}$ omitting the south pole and $\bar{V}$ omitting the north pole. Also pick $\tilde{U}$ open with $\bar{U} \subset U$, and similarly $\tilde{V}$ with $\overline{\tilde{V}} \subset V$. Pick a smooth partition of unity $\chi_{1}, \chi_{2}$ subordinate to $U, V$ such that

$$
\left.\chi_{1}\right|_{\tilde{U}}=1,\left.\chi_{2}\right|_{\tilde{V}}=1 .
$$

Further, pick $\tilde{U}_{1}, \tilde{V}_{1}$ with $\overline{\tilde{U}_{1}} \subset \tilde{U}, \overline{\tilde{V}}_{1} \subset \tilde{V}$. We may arrange things so that $\tilde{U}_{1}$, $\tilde{V}_{1}$ together still cover $S^{3}$. By the Huyghen's principle, there is a time $T_{1}>0$ such that the solution of the transformed equation (5.1) on $\left[-T_{1}, T_{1}\right] \times \tilde{U}_{1}$ only depends on the initial data restricted to $\tilde{U}$, and similarly for $\tilde{V}_{1}$. In particular, the solution of (5.1) with initial data $\left.\chi_{1} \tilde{u}\right|_{T=0},\left.\chi_{1} \tilde{u}_{T}\right|_{T=0}$ will co-incide with the original solution on $\left[-T_{1}, T_{1}\right] \times \tilde{U}_{1}$. Working in local coordinates $Y$ on $U$ and using (3.3) as well as the assumption on the initial data in Theorem 1.1, we see that Proposition 5.2 ensures the existence of a unique local solution $\tilde{u}_{U}$ of (5.1) with data $\left.\chi_{1}\left(\tilde{u},\left.\tilde{u}\right|_{T}\right)\right|_{T=0}$ on the set $\left[-T_{2}, T_{2}\right] \times U$, which will co-incide with the actual solution $\tilde{u}$ on $\left[-T_{2}, T_{2}\right] \times \tilde{U}_{1}$. Similarly, one finds a local solution $\tilde{u}_{V}$ on some set $\left[-T_{3}, T_{3}\right] \times \tilde{V}_{1}$, and these solutions necessarily co-incide on $\left[-T_{4}, T_{4}\right] \times\left(\tilde{U}_{1} \cap \tilde{V}_{1}\right)$, $T_{4}=\min \left\{T_{2}, T_{3}\right\}$. Picking a partition of unity $\tilde{\chi}_{1}, \tilde{\chi}_{2}$ subordinate to $\tilde{U}_{1}, \tilde{V}_{1}$, the expression

$$
\tilde{u}:=\tilde{\chi}_{1} \tilde{u}_{U}+\tilde{\chi}_{2} \tilde{u}_{V}
$$

is the desired solution of (5.1) on $\left[-T_{4}, T_{4}\right] \times S^{3}$. Moreover, we have

$$
\left\|\left.\left(\tilde{u}, \tilde{u}_{T}\right)\right|_{T=T_{4}}\right\|_{\left(H^{s} \times H^{s-1}\right)\left(T_{4} \times S^{3}\right)} \lesssim\left\|\left(\tilde{u}, \tilde{u}_{1}\right)\right\|_{\left(H^{s} \times H^{s-1}\right)\left(0 \times S^{3}\right)} .
$$

On can then re-iterate application of Proposition 5.2 for the initial data

$$
\left(\left.\chi_{1} \tilde{u}\right|_{T=T_{4}},\left.\chi_{1} \tilde{u}_{T}\right|_{T=T_{4}}\right),
$$

respectively

$$
\left(\left.\chi_{2} \tilde{u}\right|_{T=T_{4}},\left.\chi_{2} \tilde{u}_{T}\right|_{T=T_{4}}\right),
$$

Re-iterating this procedure finitely many times, which is possible provided the initial data norm $\left\|\left(\tilde{u}, \tilde{u}_{1}\right)\right\|_{\left(H^{s} \times H^{s-1}\right)\left(0 \times S^{3}\right)}$ is small enough, the solution is constructed on all of $(-\pi, \pi) \times S^{3}$. Indeed, according to Proposition 5.2, note that if we ensure that the initial data are small enough, such that the $H^{s}$ norm after each iteration stays below the threshold $\epsilon_{0}$, then we may always prolong the solution by a fixed amount. The solution for the main equation (1.1) is then obtained by inverting the relation (3.1).

In order to obtain the point wise bound (1.2), one uses that for $s>\frac{3}{2}$

$$
\|\tilde{u}\|_{L_{T, X}^{\infty}\left([-\pi, \pi] \times S^{3}\right)} \lesssim\|\tilde{u}\|_{L_{T}^{\infty} H_{X}^{s}\left([-\pi, \pi] \times S^{3}\right)},
$$


and we have

$$
u^{I}=\Omega(T, X) \tilde{u}^{I}
$$

where we have from [4] the relation

$$
\Omega(t, x)=\frac{2}{\sqrt{\left(1+(t-|x|)^{2}\right)\left(1+(t+|x|)^{2}\right)}}
$$

\section{REFERENCES}

[1] D. Christodoulou Global solutions of nonlinear hyperbolic equations for small initial data Comm. Pure Appl. Math. 39 (1986), no. 2, 267-282

[2] D. Geba A remark on an equation of wave maps type with variable coefficients Math. Res. Letters 16(2009), 395 - 404

[3] D. Geba, D. Tataru Gradient NLW on curved background in 4+1 dimensions Internat. Math. Res. Notices 2008 (2008), 108-58

[4] L. Hormander Lectures on Nonlinear Hyperbolic Differential Equations Mathematiques et Applications, Springer Verlag, 1997

[5] S. Klainerman The null condition and global existence to nonlinear wave equations Nonlinear systems of partial differential equations in applied mathematics, Part 1 (Santa Fe, N.M., 1984), 293-326, Lectures in Appl. Math., 23, Amer. Math. Soc., Providence, RI, 1986

[6] F. Pusateri Space-Time Resonances and the null condition for wave equations Bollettino Unione Matematica Italiana 6 (2013), no.3, 513-529

[7] F. Pusateri, J. Shatah Space-time resonances and the null condition for first order systems of wave equations Communications in Pure and Applied Mathematics 66 (2013), no. 10, 14951540.

[8] D. Tataru On global existence and scattering for the wave maps equation. Amer. J. Math. 123 (2001), no. 1, 37-77.

École Polytechnique Fédérale de Lausanne, Switzerland

E-mail address: can.gao@epfl.ch

École Polytechnique Fédérale de Lausanne, Switzerland

E-mail address: aparajita.dasgupta@epfl.ch

École Polytechnique Fédérale de Lausanne, Switzerland

E-mail address: joachim.krieger@epfl.ch 